\title{
On the Dependence between the Step Orientation and the Received Direct Solar Radiation of a PV Panel Part I: The Step Azimuthal Orientation
}

\author{
I. Visa ${ }^{1}$, D. Diaconescu ${ }^{1}$ and V. Popa ${ }^{1}$ \\ ${ }^{1} 1$ Product Design Centre for Sustainable Development, Transilvania University of Brasov \\ no. 29, Eroilor Boulevard, 500036, Brasov, Romania, tel. 0268-412088, fax 0268-410525 \\ Phone/Fax number:+0034 986 812685, e-mail: visaion@unitbv.ro,dvdiaconescu@unitbv.ro, \\ valentina.popa@unitbv.ro
}

\begin{abstract}
The approached problem regards the energetic efficiency maximization of a tracked PV panel by maximizing the received direct solar radiation, with minimum driving energy.

The objective of this first paper part is to optimize correlation between the panel received direct solar radiation and the daytime tracking steps' number, in case of an azimuthal tracked PV panel (Fig.1). The paper presents a graphical solving of the problem; from these solutions, an optimization analytic model will be developed in the future.
\end{abstract}

\section{Key words}

azimuthal tracker, step orientation/tracking, direct solar radiation, PV panel, incidence angle

\section{Introduction}

The main objective of this first part of the paper is to emphasize the dependence between the azimuthal step orientation and the received direct solar radiation of a PV panel.

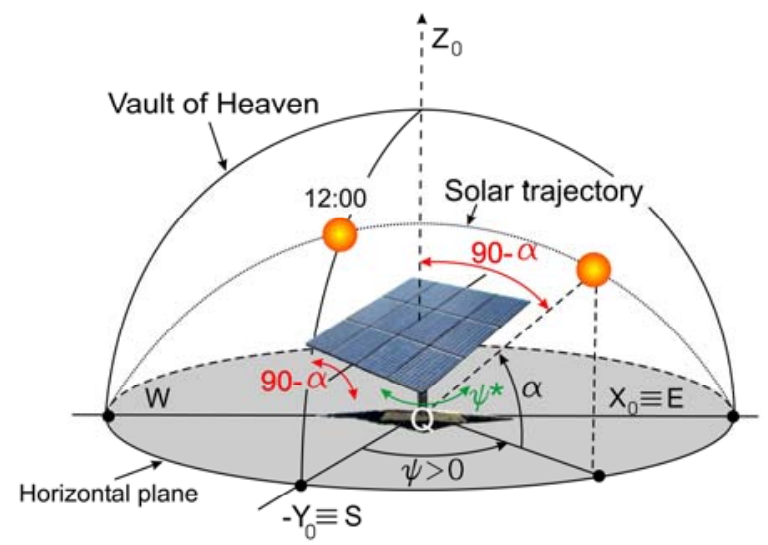

In order to do this, the paper establishes, firstly, the incidence angle sun-panel (measured between the sun-ray unit vector and the PV panel normal unit vector) and then establishes, by means of the obtained incidence angle, the $\mathrm{PV}$ received direct solar radiation.

By means of the obtained models, some nomographic charts are generated trough numerical simulations (see Fig. 2, 3, 4); for the azimuthal tracking, these charts allow graphical establishment of the parameters that assure the optimization of the correlation between the daytime (active) steps' number and the direct received solar radiation.

The obtained analytical models and the conclusions derived from the numerical simulations and graphical optimization are useful in the mathematical optimization for design of the optimal step azimuthal tracking.

In Fig. 1 there are illustrated the sun-ray angles $(\psi, \alpha)$ of the azimuthal system $\mathrm{Qx}_{0} \mathrm{y}_{0} \mathrm{Z}_{0}$ (Fig.1, a) and nearby (Fig.1, b) there is presented the geometrical scheme of the tracker based on these angles.

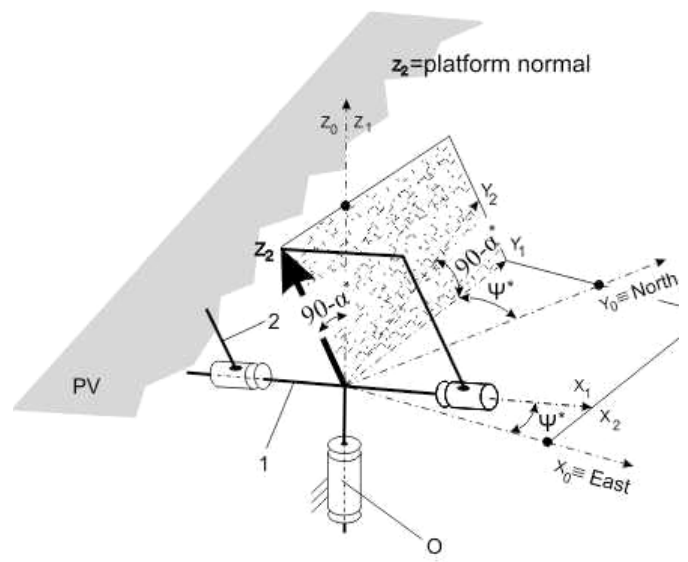

Fig. 1 The sunray angles in the azimuthal system and geometrical scheme of the azimuthal tracker. 


\section{Received Direct Solar Radiation Modelling.}

For the modeling of the direct solar radiation, received by an azimuthally tracked PV panel (Fig.1), there must be covered, previously, the next three steps: $2.1,2.2$ and 2.3.

\section{A. Unit Vectors Modelling}

Because of energetic and economical reasons, the angular displacement of the tracked PV panel is made discontinuously (in steps), so the tracker's angles have discreet variations; in order to distinguish them from the sun-ray angles (which have continuous variations: $\psi$ and $\alpha$, see Fig.1, a) in the correlations below the tracker's angles are marked with asterisk: $\psi^{*}$ and $\alpha^{*}$ (see Fig. 1, b).

Using Fig. 1, a there are established the sun-ray unit vector (1) and the PV panel normal unit vector (2):

$$
\begin{aligned}
& \bar{e}_{s r}=\left[\begin{array}{c}
\cos \alpha \cdot \sin \psi \\
-\cos \alpha \cdot \cos \psi \\
\sin \alpha
\end{array}\right]_{x_{o} y_{O} z_{o}} \\
& \bar{e}_{P V-a z}=\left[\begin{array}{c}
\sin \psi^{*} \cos \alpha^{*} \\
-\cos \psi^{*} \cdot \cos \alpha^{*} \\
\sin \alpha^{*}
\end{array}\right]_{x_{o} y_{o} z_{o}}
\end{aligned}
$$

B. Sun-PV Panel Incidence Angle on an azimuthal tracker

Using the previous unit vectors, there is modelled the incidence angle:

$$
\begin{aligned}
& \cos v=\bar{e}_{\text {sun-ray }} \cdot \bar{e}_{P V-a z}= \\
& =\cos \alpha \cdot \cos \alpha^{*} \cdot \cos \left(\psi-\psi^{*}\right)+\sin \alpha \cdot \sin \alpha^{*}
\end{aligned}
$$

\section{Direct Solar Radiation Modelling}

Using Meliss ' modelling [1], the direct solar radiation $\mathrm{R}_{\mathrm{d}}$ depends on the hour and the day of the year and it is modelled with correlation (4):

$$
\begin{aligned}
& R_{d}=1367 \cdot\left[1+0,0334 \cos \left(0,9856^{\circ} \cdot N-2,72^{\circ}\right)\right]^{*} \\
& * e^{\left(-\frac{T_{R}}{0,9+9,4 \cdot \sin \alpha}\right)}
\end{aligned}
$$

where $\mathrm{N}-$ number of the day, $\alpha$ - altitude; and $\mathrm{T}_{\mathrm{R}}-\mathrm{a}$ factor which values can be found in [1].

If the two unit vectors do not coincide, then the received direct solar radiation of the panel is modelled with correlation:

$$
R_{d r}=R_{d} \cdot \cos v
$$

where $\cos v$ is modeled by correlation (3).

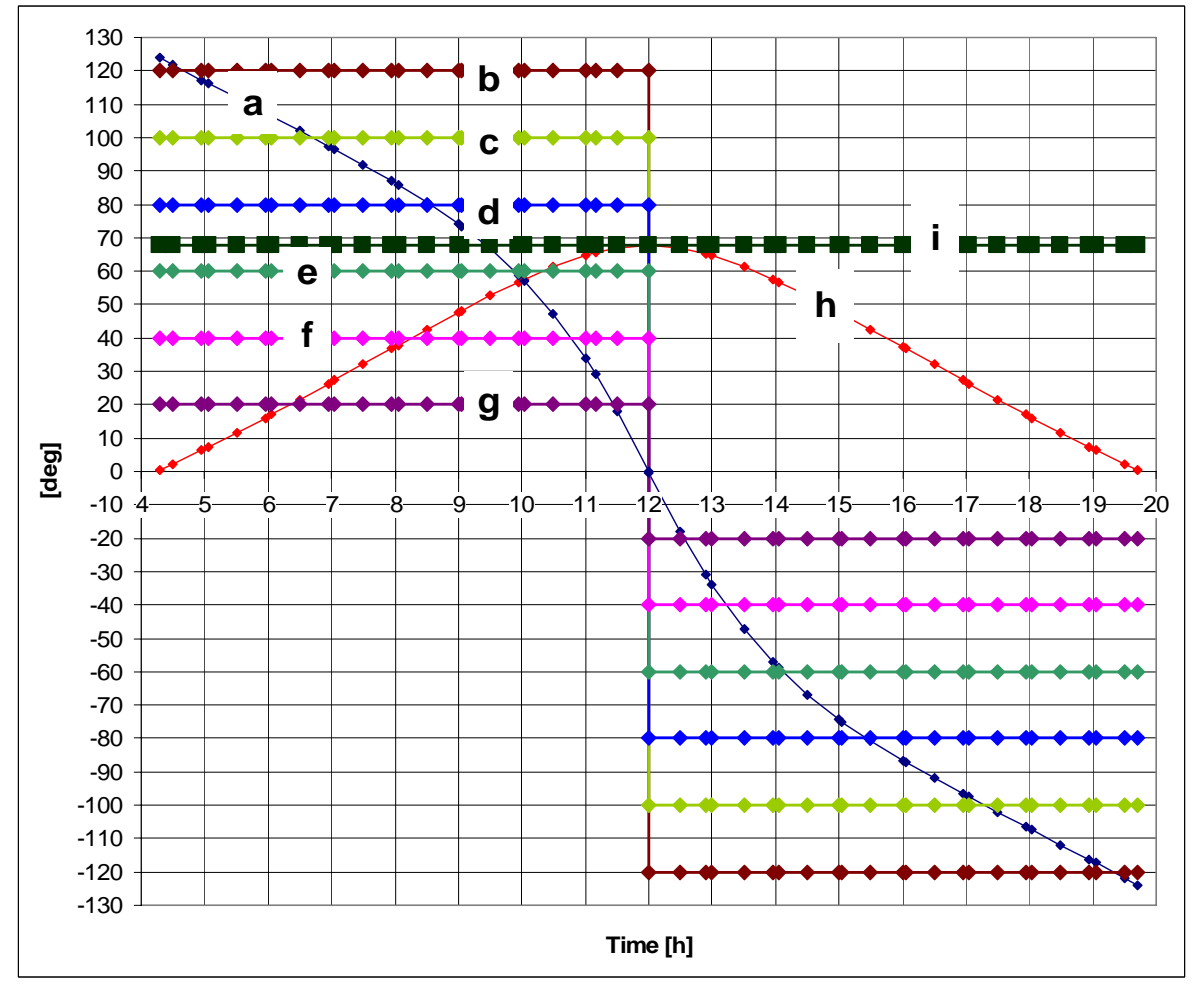

Fig. 2 Azimuth angles' variations (a-for the sunray, b,...,g-for a PV panel) and altitude angles' variations (h-for the sunray and i-for a PV panel), in the case of an azimuthal tracker, during the Summer Solstice 


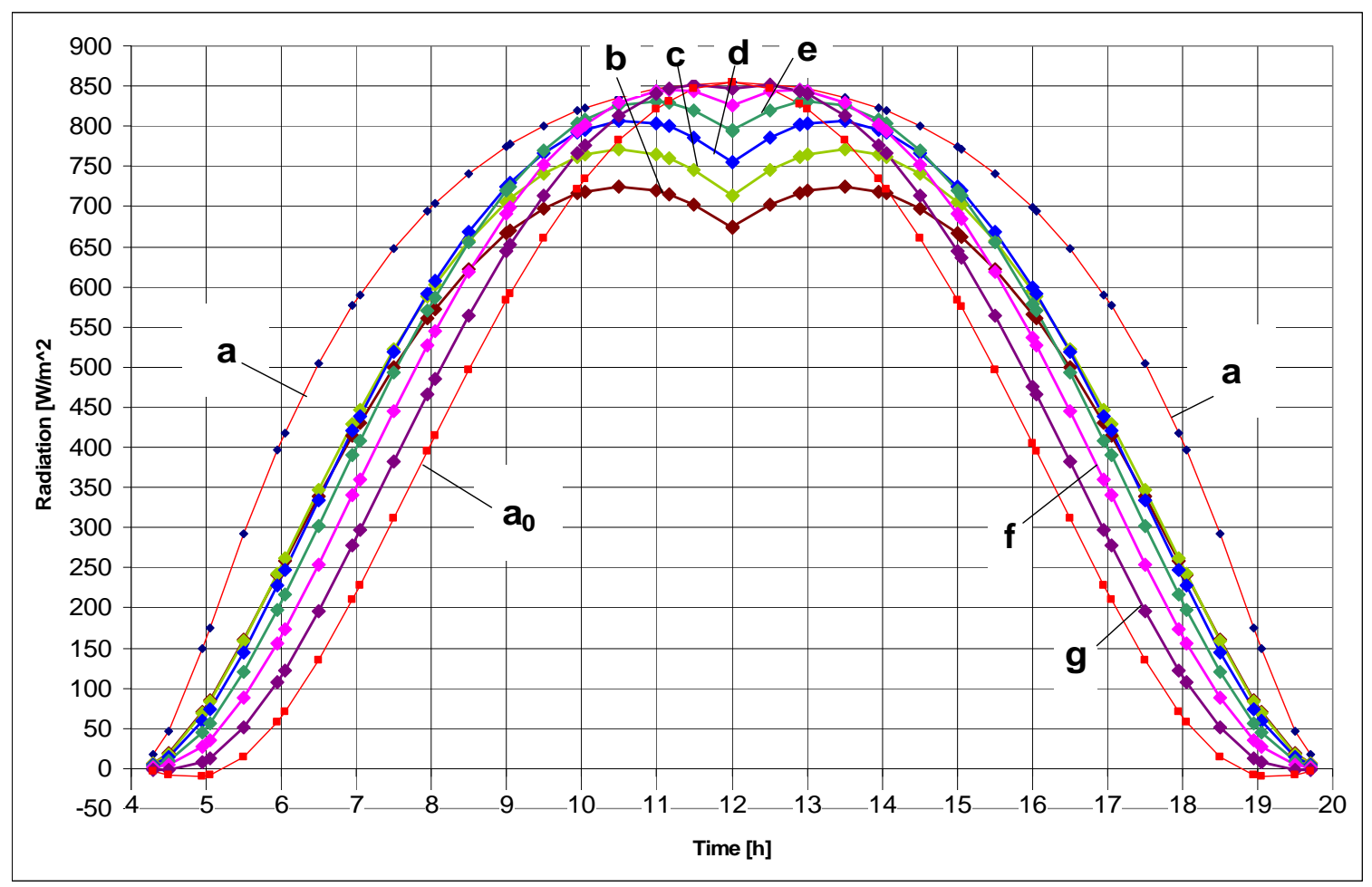

Fig. 3 Nomographic chart with variations of the solar direct radiation during the Summer Solstice (at $\alpha^{*}=68^{\circ}$ ): a- for available radiation, $\mathrm{a}_{0}$ for $\psi^{*}=0^{\circ}$ (tilted fixed PV panel), $\mathrm{b}$ for $\psi^{*}= \pm 120^{\circ}, \mathrm{c}$ for $\psi^{*}= \pm 100^{\circ}, \mathrm{d}$ for $\psi^{*}= \pm 80^{\circ}, \mathrm{e}$ for $\psi^{*}= \pm 60^{\circ}, \mathrm{f}$ for $\psi^{*}=$ $\pm 40^{\circ}, \mathrm{g}$ for $\psi^{*}= \pm 20^{\circ}$

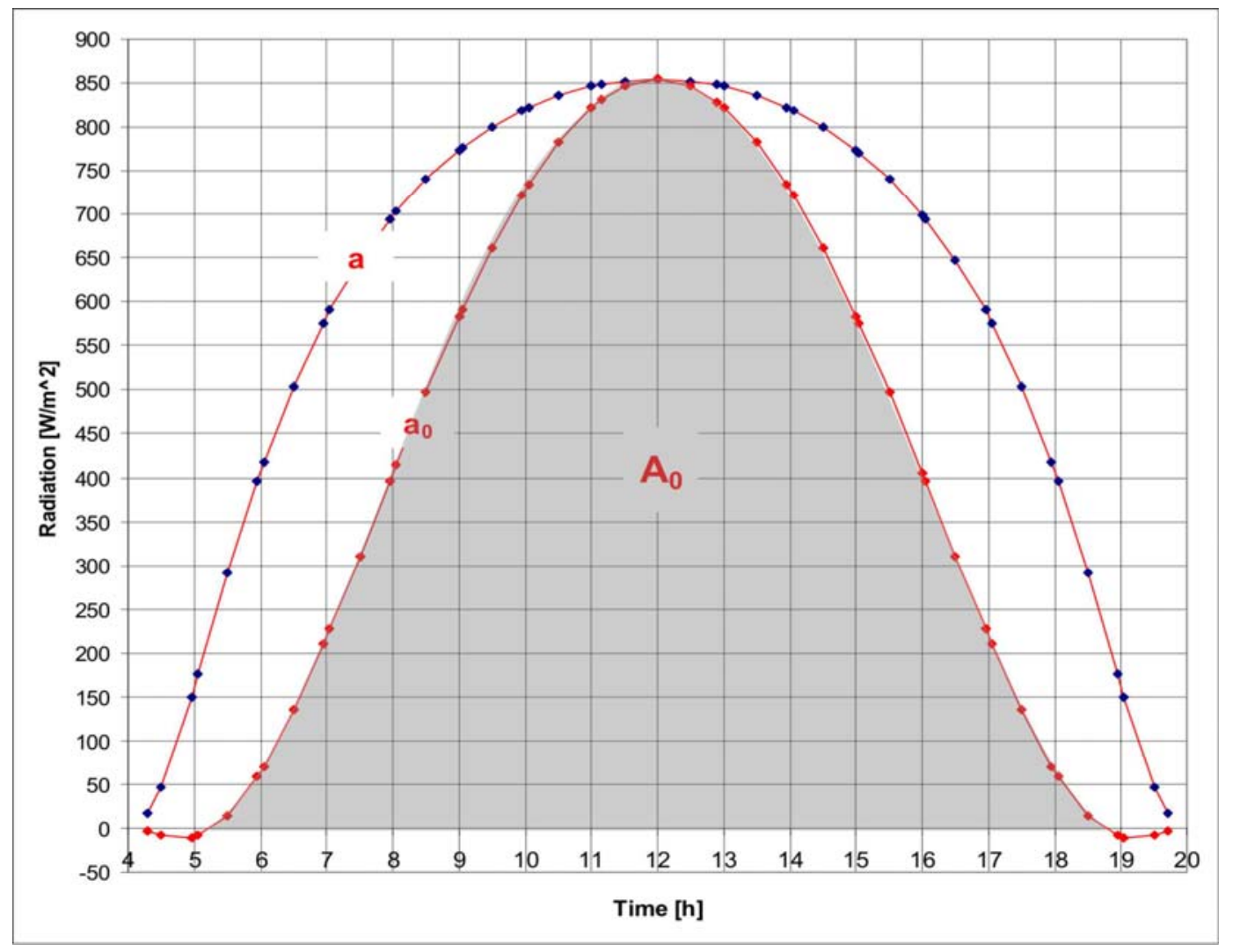

Fig. 4 Variations of the solar direct radiation during the Summer Solstice (for $\alpha^{*}=68^{\circ}$ ): for: a- available radiation, $a_{0}$ - for $\psi^{*=}$ $0^{\circ}(0$ tracking steps $=$ a tilted fixed PV panel $)$ 


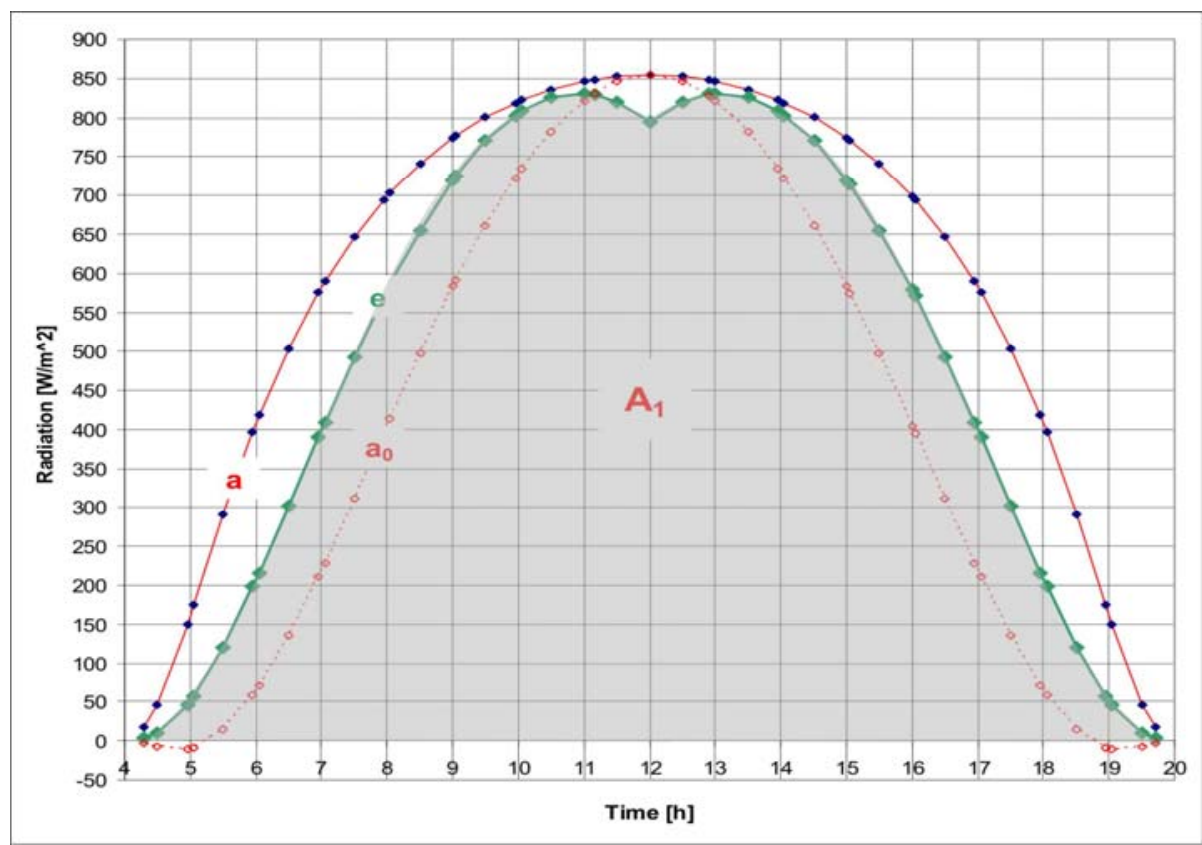

Fig. 5 Variations of the solar direct radiation during the Summer Solstice (for $\alpha^{*}=68^{\circ}$ ) at 1 tracking step: a- available radiation, $\mathrm{a}_{0^{-}}$for $\psi^{*}=0^{\circ}(0$ tracking steps $=$ a tilted fixed PV panel $)$, e- for $\psi^{*}= \pm 60^{\circ}$

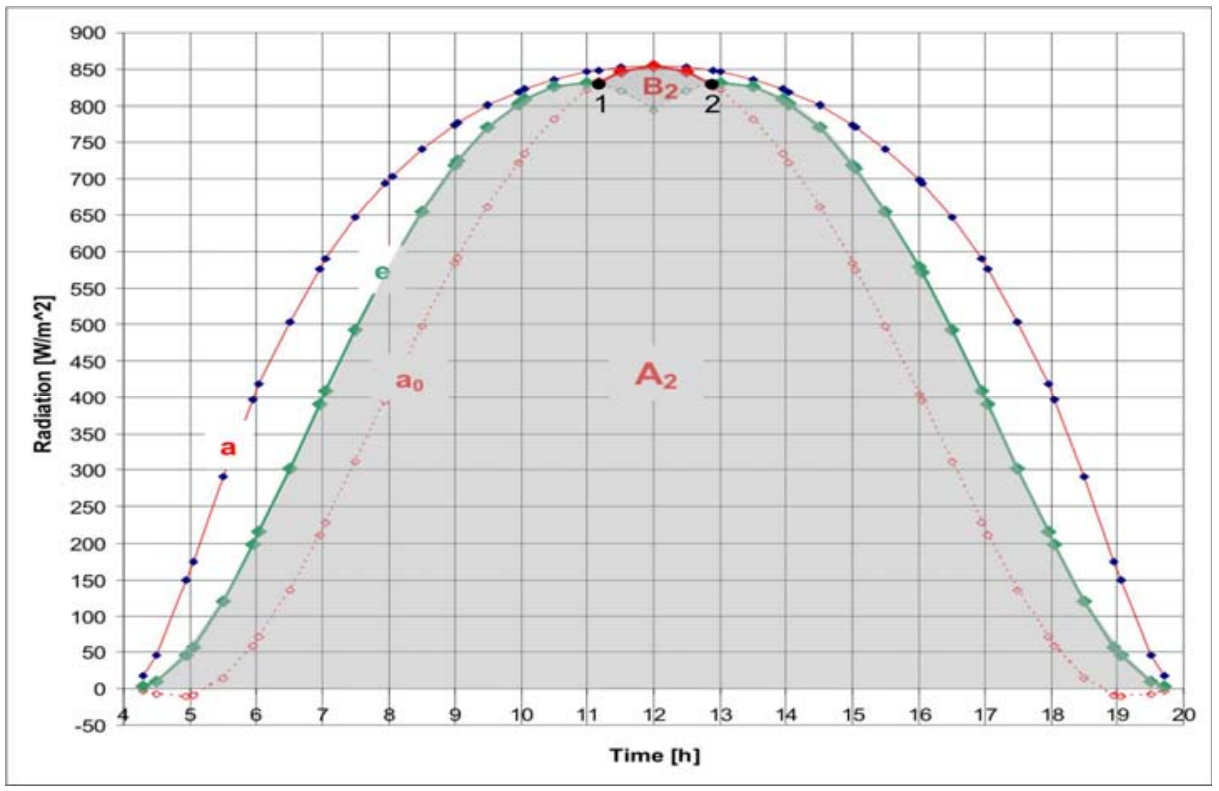

Fig. 6 Variations of the solar direct radiation during the Summer Solstice (for $\left.\alpha^{*}=68^{\circ}\right)$ at 2 steps $(1$ and 2): a- available radiation, $\mathrm{a}_{0^{-}}$for $\psi^{*}=0^{\circ}(0$ tracking steps $=$ a tilted fixed PV panel $)$, e- for $\psi^{*}= \pm 60^{\circ}$

\section{Graphical Optimization}

The optimization problem is divided in two subproblems:

a) maximization of the received direct solar radiation by optimizing the correlation between the daytime (active) steps' number and the direct received solar radiation and b) minimization of the driving energy by optimizing the tracking actuators.
This paper objective is referring only to the first aspect of the problem. For better understanding, the paper presents the graphical maximization of the received direct solar radiation.

As a numerical example, there are considered the following input data: Summer Solstice (day $N=172$, June $\left.21^{\text {st }}, \delta=+23,45^{\circ}\right)$, latitude $\varphi=45^{\circ} \mathrm{N}$, tracker altitudinal angle: $\alpha^{*}=68^{\circ}=$ const. and radiation loss coefficient $\mathrm{T}_{\mathrm{r}}$ $=4.2[1]$. 


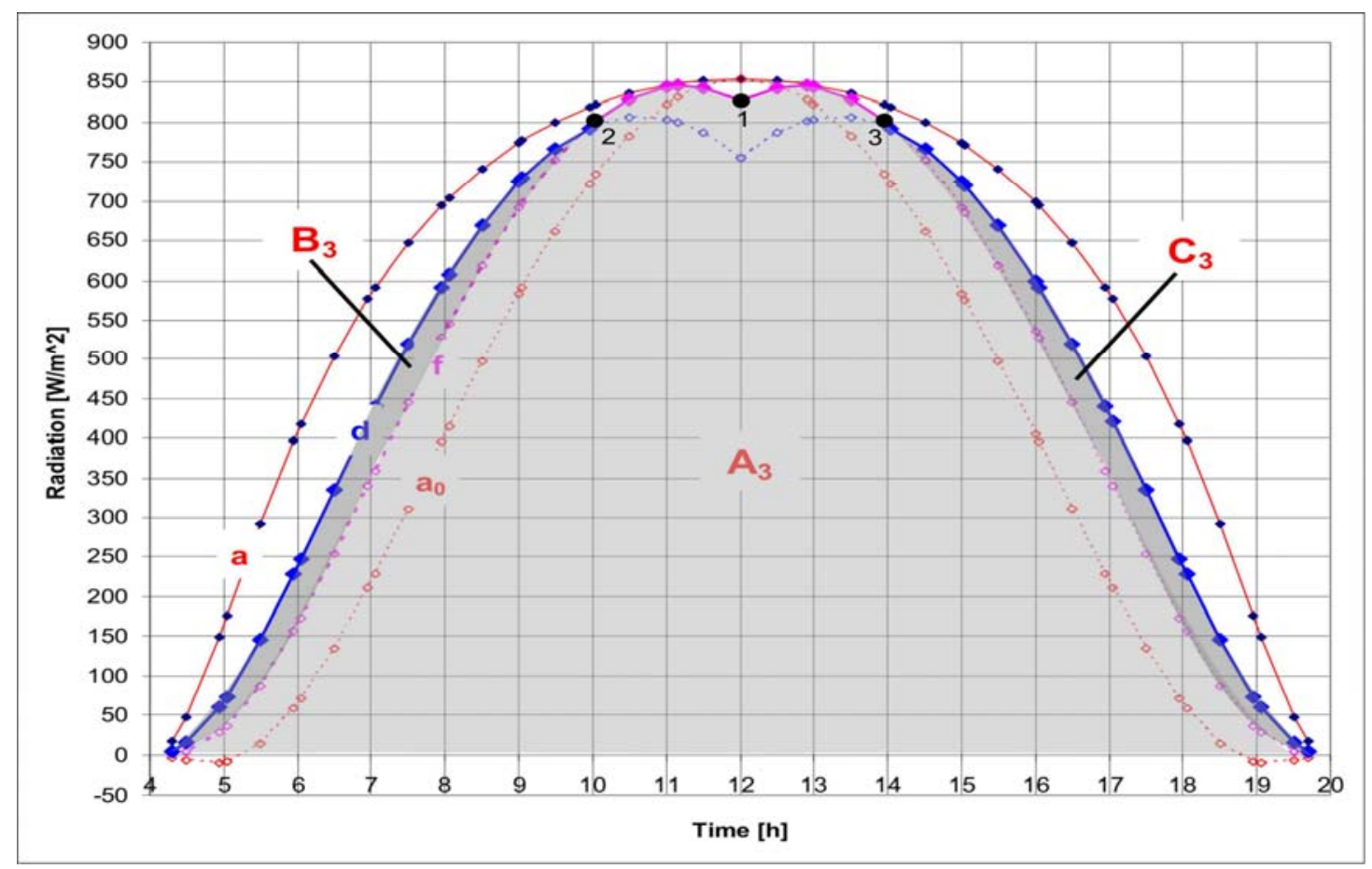

Fig. 7 Variations of the solar direct radiation during the Summer Solstice (for $\left.\alpha^{*}=68^{\circ}\right)$ at 3 steps $(1,2$ and 3): a- available radiation, $\mathrm{a}_{0}$ - for $\psi^{*}=0^{\circ}$ (tilted fixed PV panel), d- for $\psi^{*}= \pm 80^{\circ}$ and $\mathrm{f}-$ for $\psi^{*}= \pm 40^{\circ}$

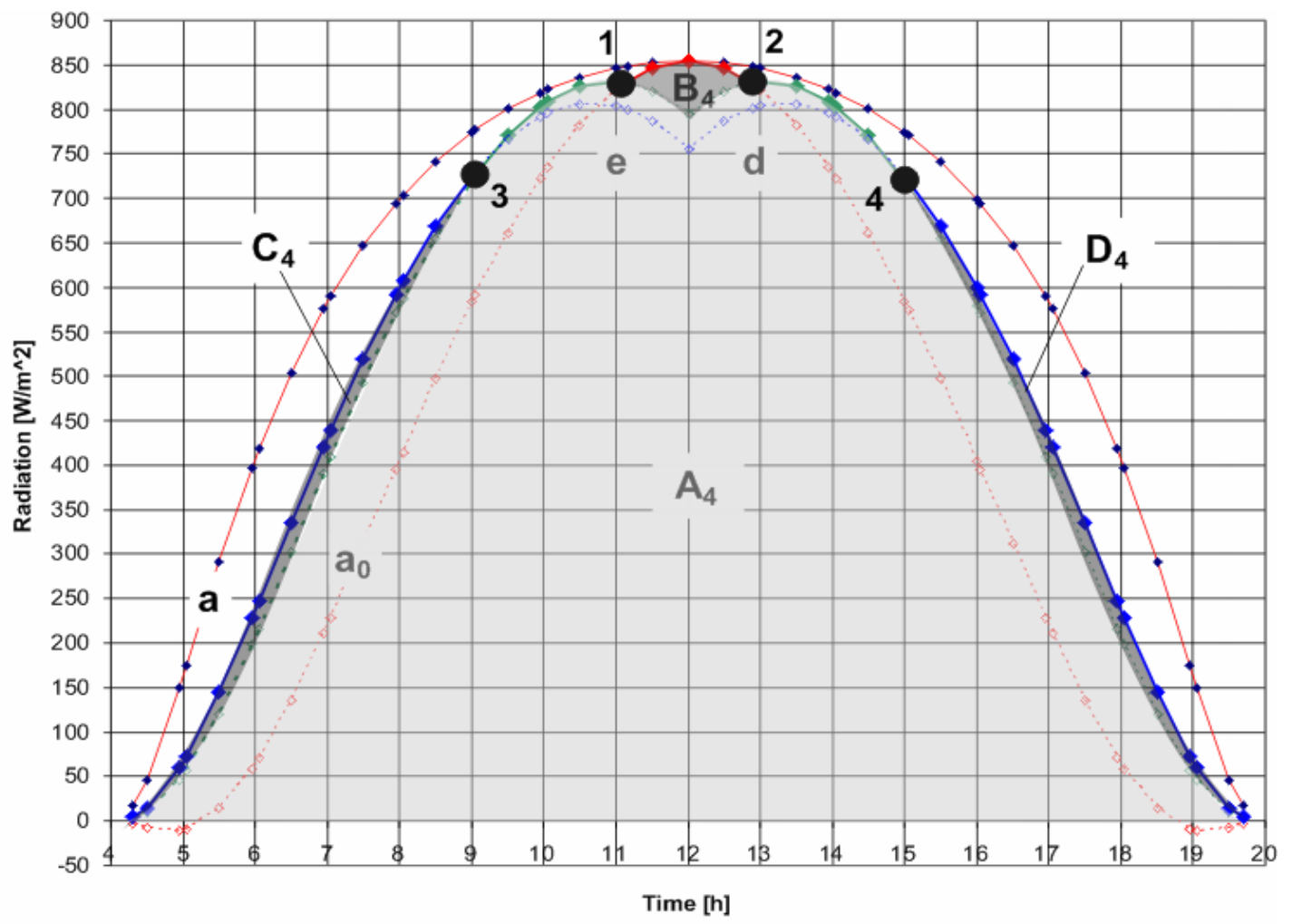

Fig. 8 Variations of the solar direct radiation during the Summer Solstice (for $\left.\alpha^{*}=68^{\circ}\right)$ at 4 steps $(1,2,3$ and 4): a- available radiation, $\mathrm{a}_{0^{-}}$for $\psi^{*}=0^{\circ}$ (tilted fixed PV panel), $\mathrm{d}$ - for $\psi^{*}= \pm 80^{\circ}$ and e- for $\psi^{*}= \pm 60^{\circ}$

Based on this input data, in Fig. 2,..,9 there is illustrated a diagram set corresponding to the discreet azimuth $\psi$ variation. This diagram set allows the graphical optimization of the correlation between the daytime step number and the direct received solar radiation, using 0,1 , 2, 3 and 4 tracking steps. If the PV panel remains fixed (0 tracking steps) at $\alpha^{*}=68^{\circ}$ and $\psi^{*}=0^{\circ}$ (see Fig. 3 and 4), the PV panel receives an energy corresponding to the $\mathrm{A}_{0}$ area (Fig. 4). When the azimuthal tracker makes a single step 1 at noon (see Fig. 3 and 5), the panel received energy, corresponding to the A1 area (Fig. 5), gets maximum for the line $\boldsymbol{e}\left(\psi^{*}= \pm 60^{\circ}\right)$ from Fig. 3 . 


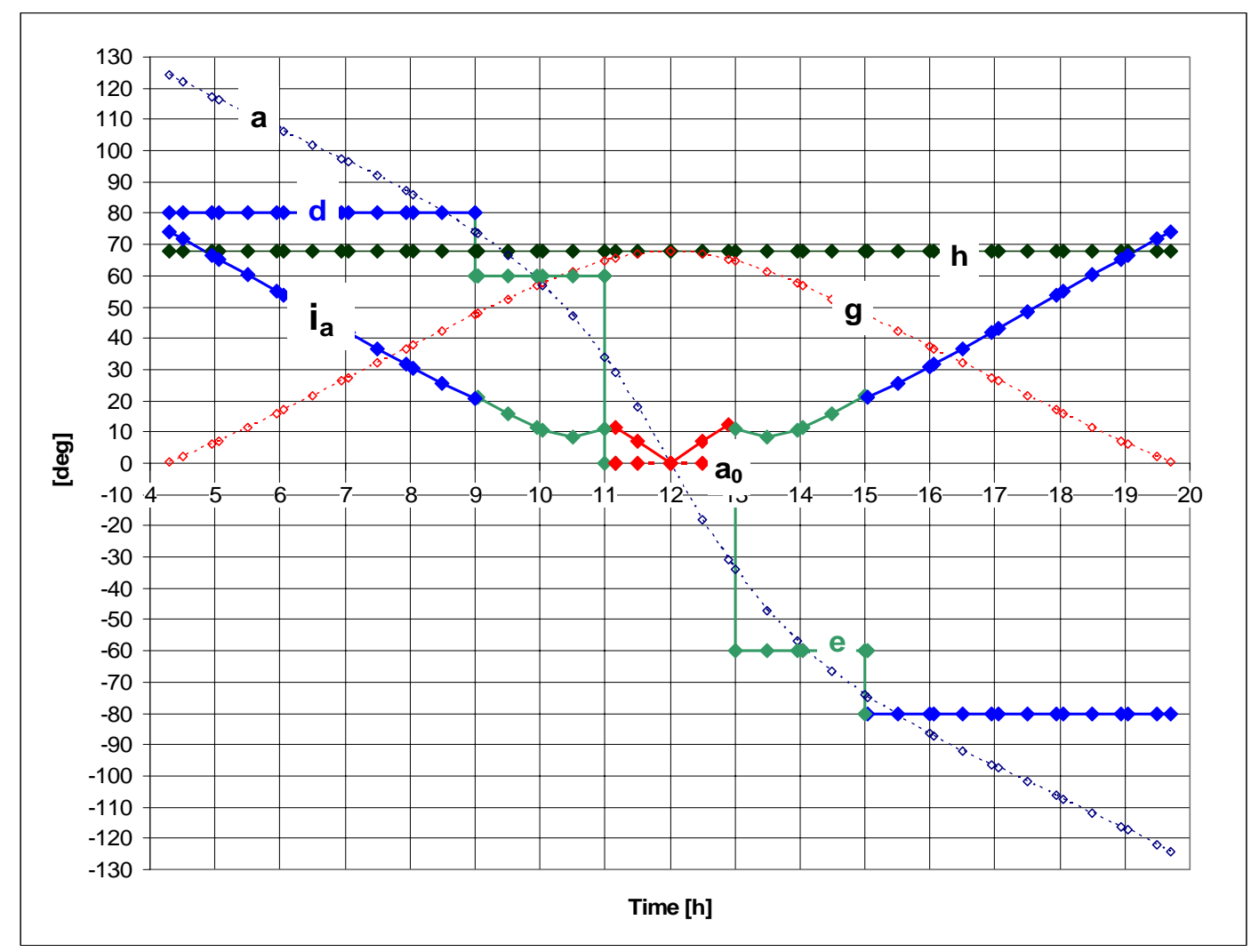

Fig. 9 Variations of the azimuth angles (a- for sunray, d, e, $a_{0}$ - for tracked panel), altitude angles (g-for the sunray and h-for a $\mathrm{PV}$ panel) and incidence angles $\left(\mathrm{i}_{\mathrm{a}}\right)$, during the Summer Solstice, for 4 steps.

So, unlike the previous case, the first step brings up moreover a panel received energy corresponding to the A1- $\mathrm{A}_{0}$ area (see Fig. 4 and 5). If the azimuthal tracker makes two steps ( 1 and 2,see Fig. 3 and 6), the panel received energy, corresponding to the $\mathrm{A} 2+\mathrm{B} 2$ area (Fig. 6), gets also maximum for the line $\boldsymbol{e}\left(\psi^{*}= \pm 60^{\circ}\right)$ from Fig. 3. So, unlike the case from Fig. 5, the two tracking steps 1 and 2 (Fig. 6) bring up moreover a panel received energy corresponding to the $\mathrm{B} 2$ area. When the azimuthal tracker makes three steps (1, 2 and 3,see Fig. 3 and 7), the panel received energy, corresponding to the $\mathrm{A} 3+\mathrm{B} 3+\mathrm{C} 3$ area (Fig. 7), gets maximum for the lines $\boldsymbol{d}$ $\left(\psi^{*}= \pm 80^{\circ}\right)$ and $\boldsymbol{f}\left(\psi^{*}= \pm 40^{\circ}\right)$ from Fig. 3. So, unlike the case from Fig. 6, the tracking steps 1, 2 and 3 (Fig. 7) bring up moreover a panel received energy corresponding to the $[(\mathrm{A} 3+\mathrm{B} 3+\mathrm{C} 3)-(\mathrm{A} 2+\mathrm{B} 2)]$ area. If the azimuthal tracker makes four steps (1, 2, 3 and 4, see Fig. 3 and 8), the panel received energy, corresponding to the $\mathrm{A} 4+\mathrm{B} 4+\mathrm{C} 4+\mathrm{D} 4$ area (Fig. 8), gets maximum for the lines $\boldsymbol{d}\left(\psi^{*}= \pm 80^{\circ}\right)$ and $\boldsymbol{e}\left(\psi^{*}= \pm 60^{\circ}\right)$ from Fig. 3. So, unlike the case from Fig. 7, the tracking steps 1,2,3 and 4 (Fig. 8) bring up moreover a panel received energy corresponding to the $[(\mathrm{A} 4+\mathrm{B} 4+\mathrm{C} 4+\mathrm{D} 4)-(\mathrm{A} 3+\mathrm{B} 3+\mathrm{C} 3)]$ area.

The sun ray azimuth and altitude variations $(\mathrm{a}, \mathrm{g})$, the azimuth and altitude variations of the PV panel $\left(\mathrm{d}, \mathrm{e}, \mathrm{a}_{0}\right.$, $h$ ), and incidence angle variations $\left(i_{a}\right)$, corresponding to the tracking in 4 steps from Fig.8, are illustrate in Fig. 9.

This cases show that the first step brings up the maximum energy, while the last step brings up the minimum energy; so, the optimal steps' number is that in which the energy brought by last step is higher than the driving energy necessary for this step.

\section{Conclusions}

From the previous analysis there can be concluded:

1) The gained energy amount per step decreases when the order number of the step increases.

2) The optimal steps' number is that in which the energy brought by last step is more than driving energy of this step.

3) According to previous conclusions, the step number choosing and the tracking programs depend on the energetic performances of the tracking actuators.

4) In the tracking program design, there must be done an optimization modelling for a big enough steps' number; this modelling will provide the angles' values $\psi_{\mathrm{j}}$ and $\alpha_{\mathrm{j}}$ which assure the maximum gained energy for the each considered step number.

5) An optimization analytic model will be developed in a following paper, using the MatLab software.

\section{References}

[1] M. Meliss, Regenerative Energiequellen, SpringerVerlag, Berlin (1997).

[2] R. Messenger and J. Ventre: Photovoltaic System Engineering, CRC Press, London (2001).

[3] D. Diaconescu a. o.: Analysis of the Sun-Earth angles used in the design of the solar collectors' trackers, in Bulletin of the Transilvania University of Brasov, Vol. 13(47), 2006, pp. 99-105. 